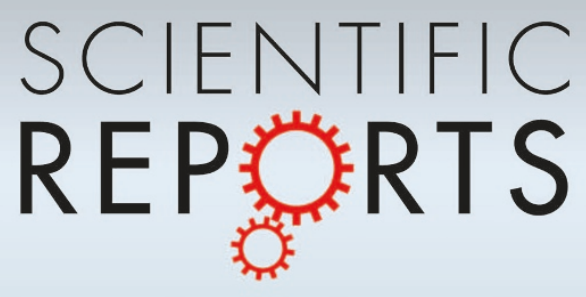

OPEN

SUBJECT AREAS:

CELL DELIVERY

NANOFABRICATION AND NANOPATTERNING

BIOSENSORS

BIOMEDICAL ENGINEERING

Received

11 December 2012

Accepted

12 July 2013

Published

26 July 2013

Correspondence and requests for materials should be addressed to

L.Y.C. (lisachen@

alumni.stanford.edu)

* These authors contributed equally to this work.

\section{Mass fabrication and delivery of 3D multilayer $\mu$ Tags into living cells}

\author{
Lisa Y. Chen ${ }^{1 *}$, Kokab B. Parizi $^{1 *}$, Hisanori Kosuge ${ }^{2}$, Kaveh M. Milaninia $^{3}$, Michael V. McConnell $^{1,2}$, \\ H.-S. Philip Wong' \& Ada S. Y. Poon'
}

'Department of Electrical Engineering, Stanford University, Stanford, CA 94305, USA, ²Division of Cardiovascular Medicine, Stanford University, CA 94305, USA, ${ }^{3}$ Department of Electrical and Computer Engineering, University of California, Santa Barbara, CA 93106, USA.

Continuous monitoring of in vivo biological processes and their evolution at the cellular level would enable major advances in our understanding of biology and disease. As a stepping stone towards chronic cellular monitoring, we demonstrate massively parallel fabrication and delivery of $3 \mathrm{D}$ multilayer micro-Tags ( $\mu$ Tags) into living cells. Both $10 \mu \mathrm{m} \times 10 \mu \mathrm{m} \times 1.5 \mu \mathrm{m}$ and $18 \mu \mathrm{m} \times 7 \mu \mathrm{m} \times 1.5 \mu \mathrm{m}$ devices containing inductive and capacitive structures were designed and fabricated as potential passive radio-frequency identification tags. We show cellular internalization and persistence of $\mu$ Tags over a 5-day period. Our results represent a promising advance in technologies for studying biology and disease at the cellular level.

○ ur ability to image living organisms has played a major role in advancing our understanding of biology and disease. However, current technology does not yet allow us to continuously monitor in vivo biological processes and their evolution at the cellular level. These limitations present major barriers to understanding biology more fully and probing the mechanisms of disease.

Considerable progress has been made in the rapidly growing field of intracellular delivery of therapeutic and imaging agents. Since the earliest discoveries of using DEAE-dextran ${ }^{1,2}$ and calcium phosphate ${ }^{3}$ as means to deliver exogenous nucleic acids to cultured mammalian cells, a multitude of chemical, physical, and viral transfection techniques have been developed to introduce drugs, proteins, and genes into living mammalian cells. More recently, researchers have studied methods to introduce bioengineered particles into cells, with diameters ranging from a few nanometers to microns $s^{4-10}$.

Simultaneously, advances in integrated circuit (IC) nanofabrication techniques have driven down the size of active devices (e.g. transistors) and embedded passive components (e.g. capacitors, inductors) on a convergence path with intracellular delivery capabilities ${ }^{11,12}$. Here we demonstrate massively parallel fabrication of 3D multilayer micro-Tags ( $\mu$ Tags) for delivery into cells and show persistence of internalized $\mu$ Tags over a 5 -day period. The design, fabrication, release, collection, delivery, and persistence of these $\mu$ Tags are presented.

\section{Results}

Design. We designed the $\mu$ Tags with passive multilayer structures suitable for use as an inductor $(\mathrm{L})$ and capacitor (C) in parallel. This is a potential platform for RFID (radio-frequency identification) tags. Figure 1 illustrates round and elongated $\mu$ Tag designs of these structures. The top two metal coil layers comprise the inductor while the bottom two metal plates separated by a high-k dielectric form a capacitive load, both of which can be varied. All metal layers are implemented with aluminum (Al) that is at least $100 \mathrm{~nm}$ thick to minimize resistance. A hafnium oxide $\left(\mathrm{HfO}_{2}\right)$ layer that is thinner than $15 \mathrm{~nm}$ is used to realize a high-k dielectric for the capacitor. The other metal layers are separated by silicon oxide $\left(\mathrm{SiO}_{2}\right)$ passivation layers that are at least $150 \mathrm{~nm}$ thick to provide sufficient isolation. The round design has lateral dimensions of $10 \mu \mathrm{m} \times 10 \mu \mathrm{m}$ while the elongated version is $18 \mu \mathrm{m} \times 7 \mu \mathrm{m}$. With at least $250 \mathrm{~nm}$ of $\mathrm{SiO}_{2}$ passivation encapsulating the device on the top and bottom side, the $\mu$ Tag has a total thickness of about $1.5 \mu \mathrm{m}$.

Mass fabrication and extraction. The $\mu$ Tags are fabricated using IC microfabrication techniques on standard silicon wafers. The bottom encapsulation $\mathrm{SiO}_{2}$ layer is grown on the silicon wafer via thermal oxidation. The bottom capacitive plate Al layer is deposited via sputtering and patterned using photolithography and dry etching. The $\mathrm{HfO}_{2}$ dielectric layer is then deposited with atomic layer depositions (ALD). The top capacitive plate Al layer is similarly deposited and patterned. The $\mathrm{HfO}_{2}$ dielectric layer is patterned using photolithography and dry 


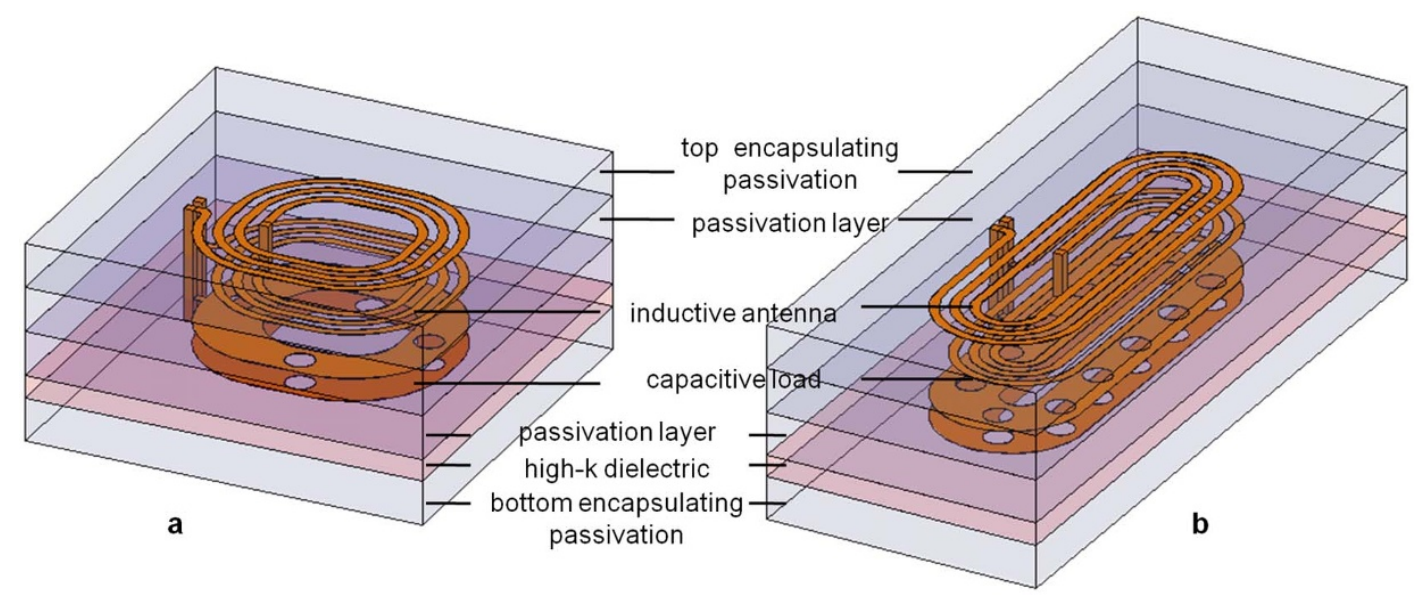

Figure 1 | Illustration of 3D $\mu$ Tag structure stack-ups for (a) round design, (b) elongated design.

etching to expose the vias holes between the bottom plate of capacitance and the first coil metal layer. The $\mathrm{SiO}_{2}$ passivation layer is deposited using low-pressure chemical vapor deposition (LPCVD) and patterned using photolithography and dry etching to expose the via holes between the top plate of capacitance and the second layer of the coil metal. Finally, the top $\mathrm{SiO}_{2}$ passivation layer is deposited to encapsulate the $\mu \mathrm{Tag}$ surface. Optical microscopy images of the wafer at various steps are given in Supplementary Figure S1.
The release process, illustrated in Figure 2, involves patterning the bottom oxide layer into wells of $\mu$ Tag devices to expose surrounding Si. Scanning electron microscopy (SEM) images of $\mu$ Tag devices prior to release are given in Figures $3 \mathrm{a}$ and $3 \mathrm{~b}$. Figures $3 \mathrm{c}$, $3 \mathrm{~d}$, and 3e show the device structure as imaged by a cross-sectional transmission electron microscopy (TEM). Exposed Si is dry etched with $\mathrm{XeF}_{2}$ to remove the layer of $\mathrm{Si}$ beneath the devices until the $\mathrm{SiO}_{2}$ encapsulated devices detach and fall into the wells. $\mathrm{XeF}_{2}$ is a vapor phase etch with an etch selectivity of silicon to photoresist, silicon

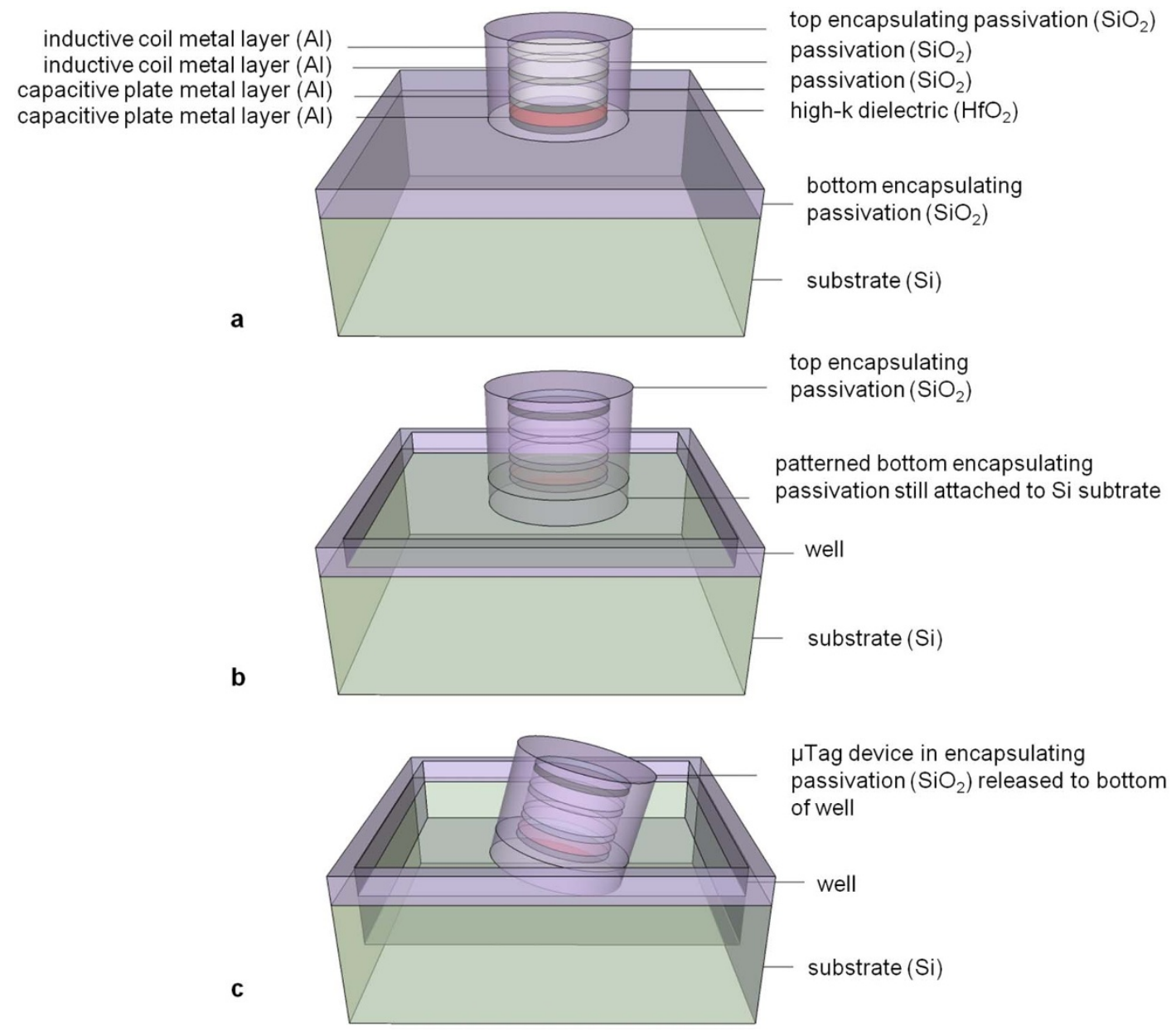

Figure $2 \mid$ Illustration of release process of single $\mu$ Tag device starting with (a) device encapsulated with top $\mathrm{SiO}_{2}$ passivation, (b) well patterning of bottom passivation layer, and (c) device released to bottom of well after Si substrate etch. 
a
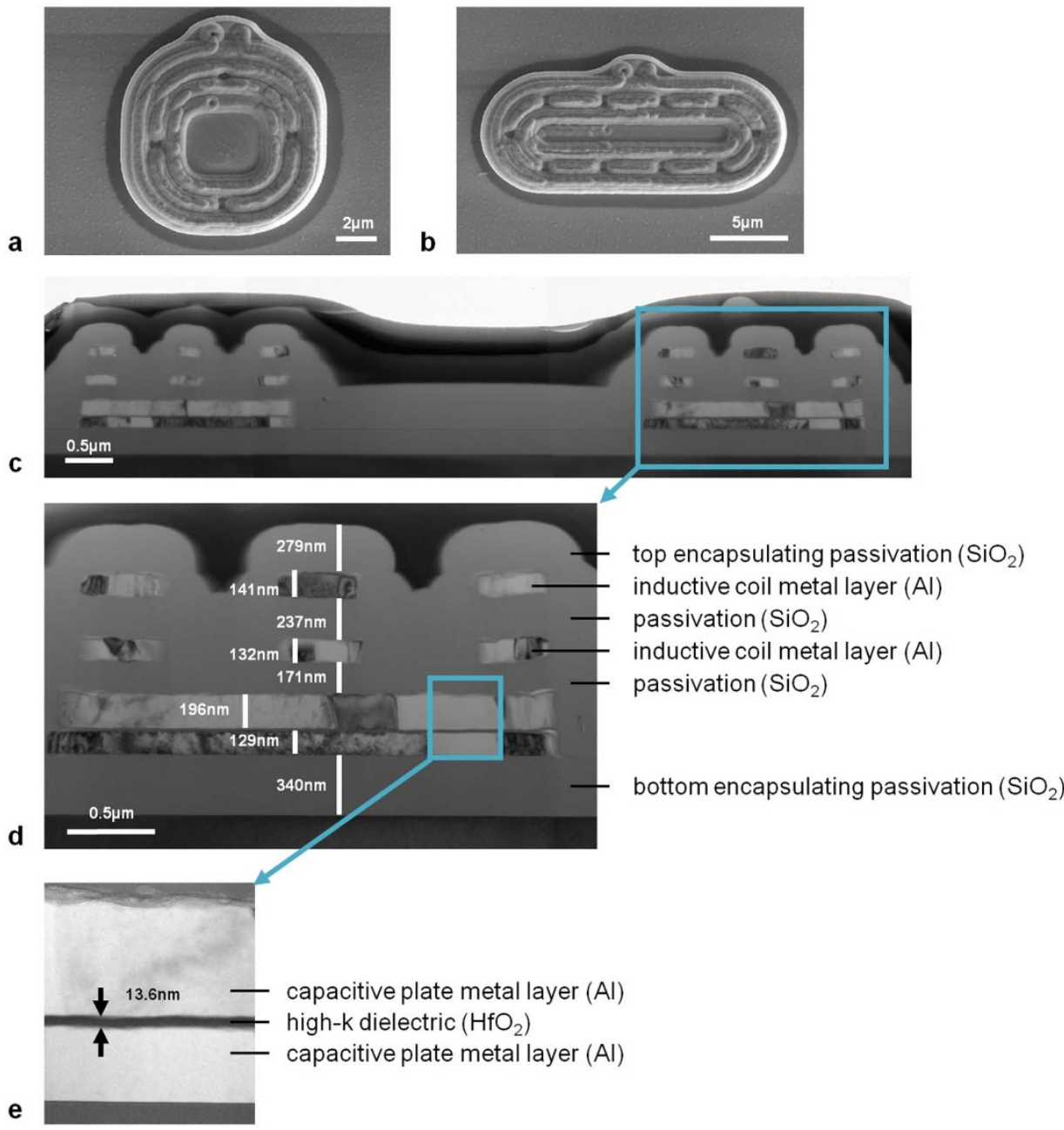

Figure 3 SEM images of $\mu$ Tag devices with (a) round design, (b) elongated design; (c) cross-section TEM image of $\mu$ Tag device structure with (d) inset showing layer stack-up and (e) further expanded view of capacitive parallel plate structure.

dioxide, and aluminum higher than 100 to 1 . Etch rate and etch profiles with this technique can be highly dependent on the substrate surface and loading effects due to sample placement. Due to the small size of $\mu$ Tags, there is a high probability that they will escape from the substrate upon release rather than fall into the wells. Hence, the number of gas flow cycles, etch time for each cycle, and the gas flow rate are carefully adjusted for each sample to prevent $\mu$ Tags from flying off the wafer in the chamber.

After the release process, $\mu$ Tag devices in individual wells are flushed with detergent-containing buffer solution to prevent aggregation and manually pipetted into centrifuge tubes. They are then spun down to concentrate the suspended solution of $\mu \mathrm{Tag}$ devices with a final concentration of about $50 \times 10^{4}$ devices per $\mathrm{mL}$.

Internationalization. Confluent cultures of mouse macrophages were incubated with $\mu$ Tags $\left(2 \times 10^{5}\right.$ cells per $\mathrm{mL}$ with a $\mu$ Tag-tocell ratio of $1: 40)$ and then imaged at regular time intervals over $30 \mathrm{~min}$ to capture internalization. Figure 4 shows time-sequence images of internalization events for both round and elongated $\mu$ Tag devices (Supplementary Videos S2 and S3). Supplementary Figure S4 shows multiple cells with internalized round and elongated $\mu$ Tag devices within the same field. Cross-sectional TEM imaging of a labeled cell in Supplementary Figure S5 further confirms internalization of the $\mu \mathrm{Tag}$ device (note some damage to the $\mu \mathrm{Tag}$ structure during manual sectioning with a diamond knife).
Qualitative biocompatibility. Confluent cultures of mouse macrophages were incubated with or without $\mu$ Tags for $24 \mathrm{~h}$. They were then washed to remove non-internalized $\mu$ Tags as well as any detached dead cells. Images of the internalized $\mu$ Tags and control (no $\mu \mathrm{Tag}$ ) cultures at day 1 are shown in Figure 5a. All cultures were subsequently maintained over the course of an additional 4 days without passaging. At the end of this 5-day experiment, cultures were again washed and imaged with a vital stain. Time-lapse image sequences of cells with $\mu$ Tags at day 5 (Supplementary Videos S6 and S7) show cell movement, further verifying cell vitality. Snapshots of internalized $\mu$ Tags and control cultures at day 5 are given in Figure 5b.

Quantitative biocompatibility. Cell viability of macrophage cultures at day 5 is quantified using a colorimetric MTS-based assay. Absorbance at the $490 \mathrm{~nm}$ wavelength is correlated with metabolic activity and thereby, the number of viable cells. Figure $5 c$ illustrates the metabolic activity in cultures with $\mu$ Tags of round and elongated designs at day 5. Data are also shown for the positive control of cell cultures incubated without $\mu$ Tags as well as negative control groups of 1 ) culture media without cells or $\mu$ Tags and 2) buffer solution containing $\mu$ Tags but no cells or media. The first negative control of culture media shows a baseline background that is consistent with a typical $0.2-0.3$ background absorbance level due to the presence of phenol red and exposure to light. Similar levels 
a

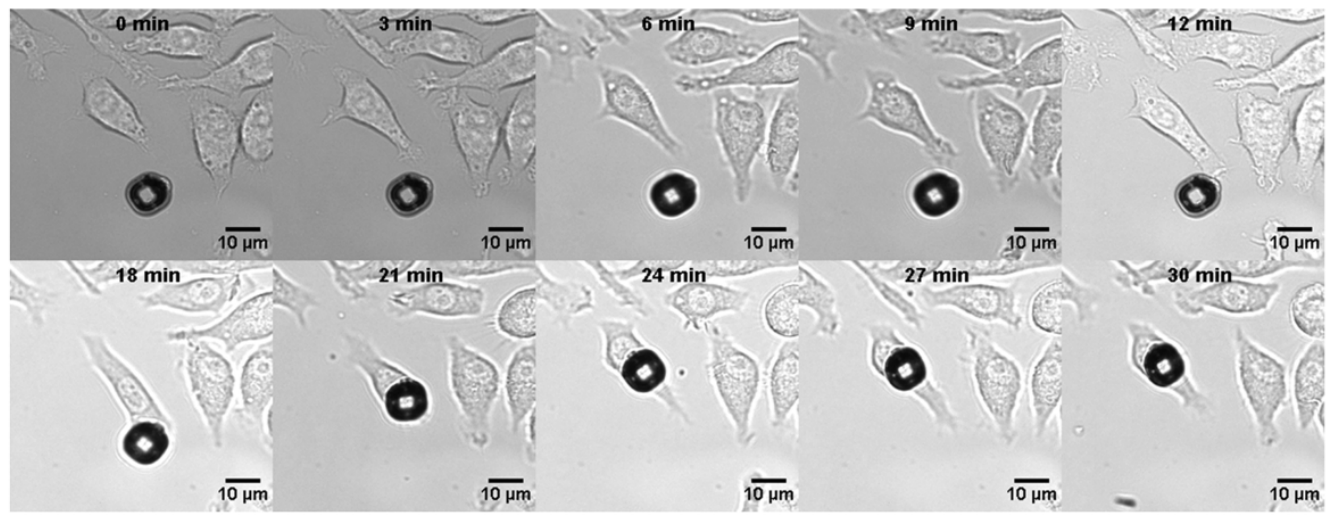

b

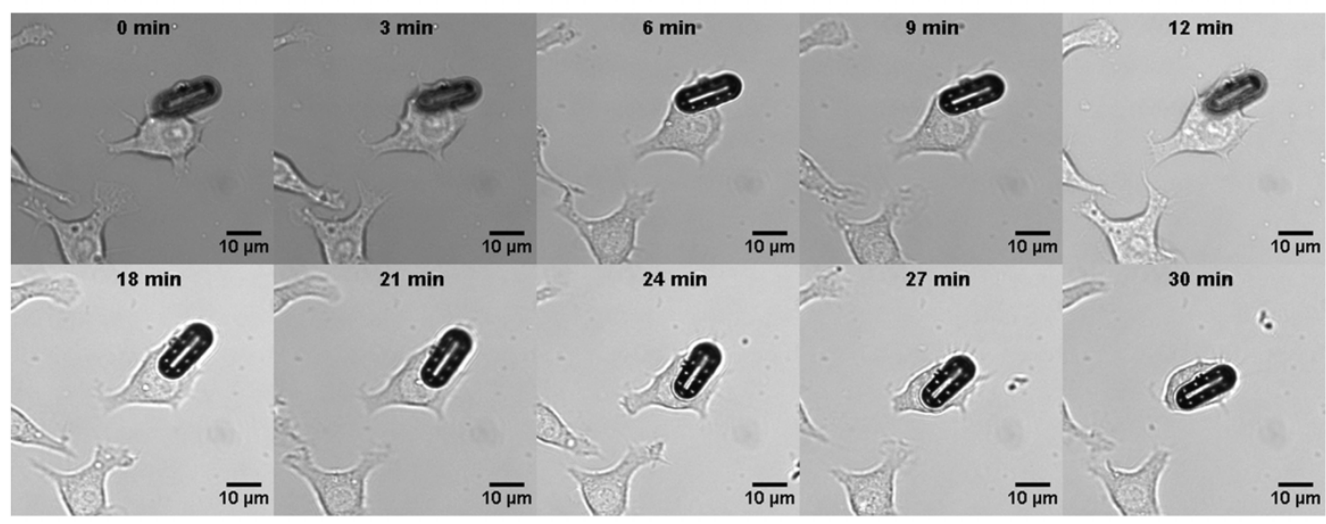

Figure 4 | Time-lapsed bright field microscopy images of cellular uptake of $\mu$ Tag device with (a) round design and (b) elongated design.

a

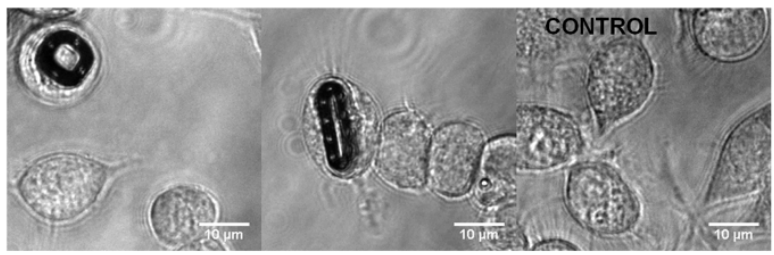

b
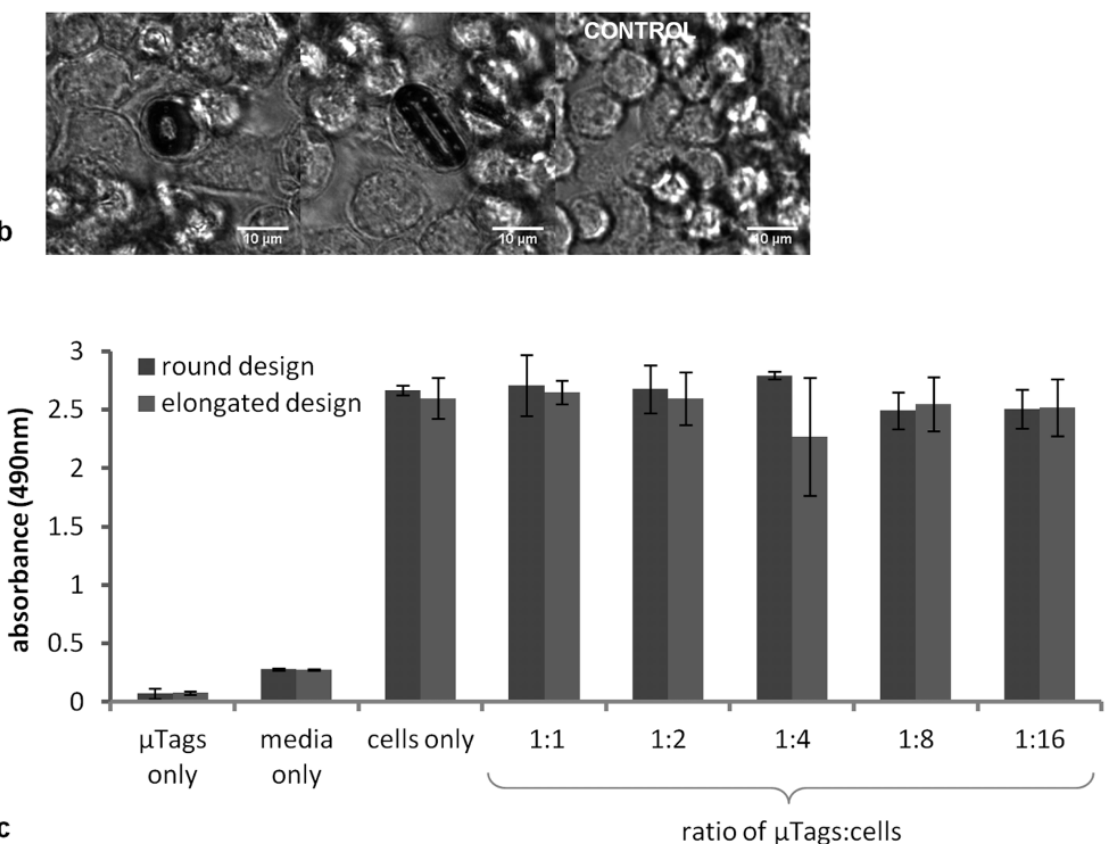

Figure $5 \mid$ Bright field microscopy image snapshots of cells with internalized round and elongated $\mu$ Tags and in the positive control group at (a) day 1 and (b) day 5 as well as (c) metabolic activity of cultures incubated with round and elongated $\mu$ Tags and in the positive and negative control groups at day 5 , as quantified by absorbance at $490 \mathrm{~nm}$ (mean \pm s.d.) with a MTS-based assay. 
of cell viability for all $\mu$ Tag-to-cell ratios (from 0 to $1: 1$ ) are seen at day 5 , with no statistically significant differences (round design: $\mathrm{p}=$ 0.3 , elongated design: $\mathrm{p}=0.7$, as determined by one-way ANOVA).

\section{Discussion}

A versatile multilayer $\mu$ Tag implementation is presented as first step towards chronic cellular monitoring with passive RFID tags. We have demonstrated mass fabrication and cell delivery of complex 3D multi-layer IC structures, not shown previously. Moreover, these internalized $\mu$ Tags are shown to persist in living cells over the course of 5 days.

Previous work has achieved delivery of single-layer, individually patterned polysilicon particles, with dimensions up to $3 \mu \mathrm{m} \times 3 \mu \mathrm{m}$ $\times 0.5 \mu \mathrm{m}^{10}$. The focused ion beam (FIB) milling they used is timeconsuming and not feasible for mass fabrication as it is limited to serial processing of individual pieces in vacuum chambers. In the present study, we have demonstrated massive parallel fabrication and delivery of large and intricate $3 \mathrm{D} \mu$ Tags with multiple metal and dielectric layers. Importantly, the IC fabrication techniques used here to create $\mu$ Tags can be extended to produce even more complex circuits for in vivo sensors.

Prior studies have shown uptake of $3 \mu \mathrm{m} \times 3 \mu \mathrm{m} \times 0.5 \mu \mathrm{m}$ polysilicon particles by phagocytic $D$. discoideum cells ${ }^{10}$ and of $10 \mu \mathrm{m} \times 6 \mu \mathrm{m} \times 0.5 \mu \mathrm{m}$ particles by macrophages ${ }^{13}$. There has also been successful introduction of unpatterned $3 \mu \mathrm{m} \times 3 \mu \mathrm{m} \times 0.5 \mu \mathrm{m}$ polysilicon particles into embryos by microinjection ${ }^{14}$. We have demonstrated uptake by macrophages of even larger cargo in the form of round $10 \mu \mathrm{m} \times 10 \mu \mathrm{m} \times 1.5 \mu \mathrm{m} \mu$ Tags as well as elongated $18 \mu \mathrm{m} \times 7 \mu \mathrm{m} \times 1.5 \mu \mathrm{m} \mu$ Tags. These macrophages typically have an average diameter of $11 \mu \mathrm{m}$ and are shown to remain viable after internationalization of these $\mu$ Tags over a 5 -day period.

We note that phagocytic cells were used to demonstrate internalization and viability. Macrophages have been shown to take up $5.5 \mu \mathrm{m}$ diameter spherical particles ${ }^{15}$, plus there are studies on the role of size and geometry of target particles on their uptake by macrophages $^{16,17}$. Interestingly, ellipsoidal particles with higher aspect ratios are shown to be more effectively phagocytosed by macrophages along their major axis. For example, macrophages with approximately $7.5 \mu \mathrm{m}$ radius spherical cell volume are able to uptake ellipsoidal particles with dimensions $3 \mu \mathrm{m} \times 3 \mu \mathrm{m} \times 14 \mu \mathrm{m}$. Other cell types may require more direct delivery methods, such as microinjection. While we have demonstrated in vitro that macrophages with internalized $\mu$ Tags can remain viable over 5 days, longer studies looking also at the effects on cell function and division, as well as cell migration and survival in vivo, will be needed.

In conclusion, we have fabricated and delivered patterned, multilayer structured $\mu$ Tags into living cells and shown cell viability over the course of 5 days. The passive LC structures formed in our 3D multilayer $\mu$ Tags can be expanded upon to build resonant RFID $\mu$ Tags for chronic cell labeling and monitoring. In particular, the capacitive load can be varied by controlling the high-k dielectric thickness. The inductor design can be also extended to comprise more layers, thereby increasing the inductive component and shifting the resonance to lower frequencies. This resonant frequency can be designed to optimize penetration of RF signal through such lossy transmission media as biological tissues ${ }^{18,19}$, and thereby improve range. Longer term, this work will open up new avenues for basic biomedical research and advances in the diagnosis and treatment of disease.

\section{Methods}

$\boldsymbol{\mu}$ Tag fabrication. To define the 500 -nm minimum feature size of the $\mu$ Tags, $\mathrm{i}$-line SPR 955 CM-0.7 resist with ASML (5500/60 model) $5: 1$ reducing stepper is used for patterning. To clearly pattern the light and dark field images, the exposure energy is adjusted to $90 \mathrm{~mJ} / \mathrm{cm}^{2}$ and $100 \mathrm{~mJ} / \mathrm{cm}^{2}$ for the capacitance and coil layers respectively. A post exposure bake of $90 \mathrm{~s}$ at $120^{\circ} \mathrm{C}$ is carried out before developing the resist.
The 10-nm $\mathrm{HfO}_{2}$ high-k dielectric layer between the capacitance plates is etched from the contact area by using the $\mathrm{Al}$ etch breakthrough recipe in Applied Materials P5000Etcher. The RF power is set to $300 \mathrm{~W}$ with gases $\mathrm{Cl}_{2}(10 \mathrm{sccm})$ and $\mathrm{BCl}_{3}$ $(40 \mathrm{sccm})$. The etch rate of $\mathrm{HfO}_{2}$ under these conditions is $10 \mathrm{~A} / \mathrm{min}$. The total etch time is $10 \mathrm{~min}$. The release step is done in Xactix e-1 using a $\mathrm{XeF}_{2}$ (xenon difluoride) isotropic silicon etcher. The number of cycles, etch time, and gas flow are individually adjusted for each sample to ensure $\mu$ Tags remain in the wells on the wafer surface upon release.

$\boldsymbol{\mu}$ Tag extraction. Manual extraction was utilized for $\mu$ Tag internalization experiments while batch extraction was used for $\mu \mathrm{Tag}$ biocompatibility experiments

Manual extraction of $\mu$ Tags from individual wells is performed by introducing about $2 \mu \mathrm{L}$ of phosphate buffered saline (PBS) with $0.05 \%$ Tween 20 . A $2-\mu \mathrm{L}$ pipette is used to agitate the suspension by pipetting up and down. The droplet is then extracted into the pipette tip and transferred into a storage tube. Repeating for 25 wells produces a $50-\mu \mathrm{L} \mu \mathrm{Tag}$ suspension with a concentration of about $5 \times 10^{5}$ devices per $\mathrm{mL}$ as confirmed through a hemocytometer count.

Batch extraction of $\mu$ Tags is achieved by rinsing the entire wafer with $10 \mathrm{~mL}$ of phosphate buffered saline (PBS) with $0.05 \%$ Tween 20 and gently sonicating the wafer in solution for $5 \mathrm{~min}$ at power level 5 on a VWR 50D Ultrasonic Cleaner. The resulting suspension is transferred into a centrifuge tube. Spinning down at a speed of $1500 \mathrm{rpm}(400 \times \mathrm{g})$ for $5 \mathrm{~min}$ and removing the supernatant reduces the solution down to a volume of $50 \mu \mathrm{L}$ with a concentration of about $5 \times 10^{5}$ devices per $\mathrm{mL}$ as confirmed through a hemocytometer count.

Cells. The mouse leukemic monocyte macrophage cell line RAW 264.7 (Abcam) is used. They are reconstituted and maintained in Dulbecco's modified Eagle's Medium (DMEM) containing 10\% fetal bovine serum (FBS) under standard culture conditions $\left(37^{\circ} \mathrm{C}, 5 \% \mathrm{CO}_{2}\right.$, humidified) until confluent.

$\boldsymbol{\mu}$ Tag internalization. The Bioptechs Delta $\mathrm{T}$ controlled dish system is used to maintain cultures at $37^{\circ} \mathrm{C}$ over the observation period on $35 \mathrm{~mm}$ outer diameter Delta T3 dishes. In internalization studies, suspended $\mu$ Tags in PBS Tween 20 are added to the cell culture with a concentration of $2 \times 10^{5}$ cells per $\mathrm{mL}$ to produce a $\mu$ Tag-to-cell ratio of $1: 40$ in a final volume of $1.1 \mathrm{~mL}$. Time-lapse video microscopy is performed using a DeltaVision wide-field microscope under bright field illumination with images collected at $30 \mathrm{~s}$ intervals for $30 \mathrm{~min}$. Cross-sectional images of the sample confirming internalization of $\mu$ Tags are produced by varying depth of focus by $0.5 \mu \mathrm{m}$ increments.

Samples for TEM sectioning were prepared by incubating a confluent culture of mouse macrophages in the presence of $\mu$ Tags on 8 -well Lab-Tek chambered coverglass. There is a concentration of $1 \times 10^{6}$ cells per $\mathrm{mL}$ with a $\mu$ Tag-to-cell ratio of $1: 2$ in a final volume of $250 \mu \mathrm{L}$ per well. After $24 \mathrm{~h}$ incubation period under standard conditions $\left(37^{\circ} \mathrm{C}, 5 \% \mathrm{CO}_{2}\right.$, humidified), the samples are fixed in $2 \%$ glutaraldehyde and $4 \% \mathrm{p}$-formaldehyde in $0.1 \mathrm{M}$ HEPES buffer ( $\mathrm{pH}$ 7.2) for $20 \mathrm{~min}$ at room temperature. These samples are subsequently postfixed in $1 \%$ osmium tetroxide $\left(\mathrm{OsO}_{4}\right)$, rinsed with double-distilled $\mathrm{H}_{2} \mathrm{O}$, stained with uranyl acetate, dehydrated in a graded ethanol series, then infiltrated and embedded in Epon. The samples are manually sectioned with a diamond knife and the sections are finally imaged with a Jeol 1230 TEM.

$\boldsymbol{\mu}$ Tag qualitative biocompatibility. Cultured cells with a concentration of $1 \times 10^{5}$ cell per $\mathrm{mL}$ are incubated in the presence of $\mu$ Tag devices on 2-well Lab-Tek chambered coverglass at a $\mu$ Tag-to-cell ratio of $1: 20$ in a final volume of $500 \mu \mathrm{L}$ per well. The cells of the same concentration are cultured in the absence of $\mu$ Tag devices in the control group. All cultures are imaged using a DeltaVision wide-field microscope under bright field illumination. Cross-sectional images of the sample confirming internalization of $\mu$ Tags are produced by varying depth of focus by $0.5 \mu \mathrm{m}$ increments. In addition to cross-sectional imaging, time-lapse video microscopy is used to capture cell movement every $5 \mathrm{~s}$ over 3 -min periods. Trypan blue is used as a vital stain to corroborate cell viability. Cultures are maintained under standard conditions $\left(37^{\circ} \mathrm{C}, 5 \% \mathrm{CO}_{2}\right.$, humidified) without passaging between observations.

$\boldsymbol{\mu}$ Tag quantitative biocompatibility. Confluent cultures of macrophages with a fixed concentration of $1 \times 10^{5}$ per mL cells are incubated with $10 \mu \mathrm{L}$ of a suspended $\mu \mathrm{Tag}$ solution for a final volume of $100 \mu \mathrm{L}$ per well on a 96-well microplate. A $1: 2$ dilution series of a suspended solution of round $\mu$ Tags with a starting concentration of $1 \times 10^{6}$ devices per $\mathrm{mL}$ systematically varies the number of $\mu$ Tags in each well to form $\mu$ Tagto-cell ratios of $1: 1,1: 2,1: 4,1: 8$, and $1: 16$. This is then repeated for elongated $\mu$ Tags and both sets of samples are created at all concentrations in triplicates. Two sets of triplicate positive control samples consist of a $10 \mu \mathrm{L}$ PBS Tween 20 solution added to a confluent culture of macrophages at a concentration $1 \times 10^{5}$ cells per $\mathrm{mL}$ in a final volume of $100 \mu \mathrm{L}$ per well. Two sets of triplicate negative controls contain $10 \mu \mathrm{L}$ PBS Tween 20 solution and $90 \mu \mathrm{L}$ of cell media (DMEM with 10\% FBS) without cells or $\mu$ Tags. We also include additional negative controls in the form of sensors in a $100 \mu \mathrm{L}$ PBS Tween 20 solution without media at the same $1 \times 10^{4}$ per $\mathrm{mL}$ concentration as in the $1: 1$ wells.

Cultures are maintained under standard conditions $\left(37^{\circ} \mathrm{C}, 5 \% \mathrm{CO} 2\right.$, humidified) without passaging. At day 5, we use the MTS-based CellTiter 96 Aqueous One Solution Cell Proliferation Assay to characterize cell viability. $20 \mu \mathrm{L}$ of the MTS and PES containing reagent is added to each well and incubated under standard conditions $\left(37^{\circ} \mathrm{C}, 5 \% \mathrm{CO}_{2}\right.$, humidified) for $4 \mathrm{~h}$. The plate is then measured with 
SpectraMax M2 microplate reader. Three measurements of absorbance at $490 \mathrm{~nm}$ are read and averaged. Wells with only PBS solution without assay reagent are used as a blank.

1. Vaheri, A. \& Pagano, J. S. Infectious poliovirus RNA: A sensitive method of assay. Virology 27, 434-436 (1965).

2. McCutchan, J.H. \& Pagano, J. S. Enhancement of the infectivity of simian virus 40 deoxyribonucleic acid with diethylaminoethyl-dextran. J. Natl. Cancer Inst. 41, 351-357 (1968).

3. Graham, F. L. \& van der Eb, A. J. A new technique for the assay of infectivity of human adenovirus 5 DNA. Virology 52, 456-67 (1973).

4. Levy, R., Shaheen, U., Cesbron, Y. \& See, V. Gold nanoparticles delivery in mammalian live cells: a critical review. Nano Reviews 1, 4889 (2010).

5. Akin, D. et al. Bacteria-mediated delivery of nanoparticles and cargo into cells. Nat. Nanotechnol. 2, 441-449 (2007).

6. Soman, N. R., Marsh, J. N., Lanza, G. M. \& Wickline, S. A. New mechanisms for non-porative ultrasound stimulation of cargo delivery to cell cytosol with targeted perfluorocarbon nanoparticles. Nanotechnology 19, 185102 (2008).

7. Lin, J. et al. Rapid delivery of silver nanoparticles into living cells by electroporation for surface-enhanced Raman spectroscopy. Biosens. Bioelectron. 25, 388-394 (2009).

8. Seo, W. S. et al. FeCo/graphitic-shell nanocrystals as advanced magneticresonance-imaging and near-infrared agents. Nat. Mater. 5, 971-976 (2006).

9. Terashima, M. et al. Human ferritin cages for imaging vascular macrophages. Biomaterials 32, 1430-1437 (2011).

10. Gomez-Martinez, R. et al. A. Intracellular silicon chips in living cells. Small 6, 499-502 (2010).

11. Bohr, M. The evolution of scaling from the homogeneous era to the heterogeneous era. IEEE Int. Elec. Dev. Mtg., 1.1.1-1.1.6 (2011).

12. Lee, B. \& Wong, H.-S. P. NiO resistance change memory with a novel structure for 3D integration and improved confinement of conduction path. Proc. Int. Symp. VLSI Techol., 28-29 (2009).

13. Fernandez-Rosas, E. et al. Intracellular polysilicon barcodes for cell tracking. Small 5, 2433-2439 (2009).

14. Fernandez-Rosas, E. et al. Internalization and cytotoxicity analysis of siliconbased microparticles in macrophages and embryos. Biomed. Microdevices $\mathbf{1 2}$ 371-379 (2010).

15. Gonzalez, O., Smith, R. L. \& Goodman, S. B. Effect of size, concentration, surface area, and volume of polymethylmethacrylate particles on human macrophages in vitro. J. Biomed. Mater. Res. 30, 463-473 (1996).

16. Champion, J. A. \& Mitragotri, S. Role of target geometry in phagocytosis. Proc. Natl. Acad. Sci. U.S.A. 103, 4930-4934 (2006).
17. Champion, J. A., Walker, A. \& Mitragotri, S. Role of particle size in phagocytosis of polymeric microspheres. Pharm. Res. 25, 1815-1821 (2008).

18. Poon, A. S. Y., O’Driscoll, S. \& Meng, T. H. Optimal frequency for wireless power transmission into dispersive tissue. IEEE Trans. Ant. Propag. 58, 1739-1750 (2010).

19. Kim, S., Ho, J. S., Chen, L. Y. \& Poon, A. S. Y. Wireless power transfer to a cardiac implant. Appl. Phys. Lett. 101, 073702 (2012).

\section{Acknowledgements}

We thank Jon Mulholland, John Perrino, and Lydia-Marie Joubert of the Cell Sciences Imaging Facility at Stanford for their expert advice and technical assistance. We also thank Provine and $\mathrm{He}$ (Linda) Yi for assistance in SEM and TEM analysis and discussions about FIB during the course of this work. This work is supported in part by the Seed Grant of the Stanford Center for Integrated Systems and the W.M. Keck Foundation through the W.M. Keck Foundation Faculty Scholar Award to H.-S.P.W. Fabrication of the $\mu$ Tag was performed at the Stanford Nanofabrication Facility, a node of the National Science Foundation funded National Nanotechnology Infrastructure Network (NNIN).

\section{Author contributions}

L.Y.C., M.V.M., H.-S.P.W. and A.S.Y.P. conceived the experiments. L.Y.C. and K.B.P. designed the $\mu$ Tag devices, K.B.P. fabricated them, L.Y.C. extracted them, and H.K. cultured the cells. K.M. provided input on the fabrication and extraction techniques. L.Y.C. and H.K. designed and performed the internationalization and biocompatibility experiments. L.Y.C and K.B.P. analyzed the data and wrote the paper. All authors reviewed and critiqued the results, and made key revisions to the manuscript. Correspondence and requests for materials should be addressed to L.Y.C.

\section{Additional information}

Supplementary information accompanies this paper at http://www.nature.com/ scientificreports

Competing financial interests: The authors declare no competing financial interests. How to cite this article: Chen, L.Y. et al. Mass fabrication and delivery of 3D multilayer $\mu$ Tags into living cells. Sci. Rep. 3, 2295; DOI:10.1038/srep02295 (2013).

(i) $\Theta$ This work is licensed under a Creative Commons Attributionvisit http://creativecommons.org/licenses/by-nc-nd/3.0 